\title{
Implementing low level features for human aggressive movement detection
}

\begin{abstract}
In this real world, being able to identify the signs of imminent abnormal behaviors such as aggression or violence and also fights, is of extreme importance in keeping safe those in harm's way. This research propose an approach to figure out human aggressive movements using Horn-Schunck optical flow algorithm in order to find the flow vector for all video frames. The video frames are collected using digital camera. This research guides and discovers the patterns of body distracted movement so that suspect of aggression can be investigated without body contact. Using the vector of this method, the abnormal and normal video frames are then classified and utilized to define the aggressiveness of humans. Preliminary experiment result showed that the low level of feature extraction can classify human aggressive and non-aggressive movements.
\end{abstract}

Keyword: Optical flow; Horn-Schunck algorithm; Aggressive movement; Non-aggressive movement 